\title{
GMR
}

\section{Molecular cloning and expression analysis of jasmonic acid dependent but salicylic acid independent LeWRKY1}

M. Lu' ${ }^{1}$, L.F. Wang ${ }^{3}$, X.H. Du ${ }^{3}$, Y.K. Yu², J.B. Pan', Z.J. Nan', J. Han ${ }^{1}$, W.X. Wang ${ }^{1}$, Q.Z. Zhang ${ }^{1}$ and Q.P. Sun ${ }^{1}$

${ }^{1}$ Plant Science and Technology College, Beijing University of Agriculture, Beijing, China

${ }^{2}$ Center of Tissue Culture, Beijing University of Agriculture, Beijing, China

${ }^{3}$ School of Life Sciences, Shandong Normal University, Jinan, China

Corresponding author: Q.P. Sun

Email: sunqp@bac.edu.cn

Genet. Mol. Res. 14 (4): 15390-15398 (2015)

Received February 4, 2015

Accepted May 28, 2015

Published November 30, 2015

DOI http://dx.doi.org/10.4238/2015.November.30.16

ABSTRACT. Various plant genes can be activated or inhibited by phytohormones under conditions of biotic and abiotic stress, especially in response to jasmonic acid (JA) and salicylic acid (SA). Interactions between JA and SA may be synergistic or antagonistic, depending on the stress condition. In this study, we cloned a full-length cDNA (LeWRKY1, GenBank accession No. FJ654265) from Lycopersicon esculentum by rapid amplification of cDNA ends. Sequence analysis showed that this gene is a group II WRKY transcription factor. Analysis of LeWRKY1 mRNA expression in various tissues by qRT-PCR showed that the highest and lowest expression occurred in the leaves and stems, respectively. In addition, LeWRKY1 expression was induced by JA and Botrytis cinerea Pers., but not by SA.

Key words: LeWRKY1; Jasmonic acid; Salicylic acid; Botrytis cinerea 


\section{INTRODUCTION}

Plants are typically sessile organisms, and therefore, cannot avoid the stressor when under biotic or abiotic stress. They have thus evolved the ability to respond to stress stimuli in a timely and specific manner. When exposed to stress, a set of genes can be activated or inhibited. These changes in gene expression are responsible for some of the specific responses observed under conditions of biotic and abiotic stress. The products of these genes are generally regulatory proteins, which may subsequently regulate cell responses and activate the expression of quick response defense genes. A transcription factor, WRKY, is strongly and rapidly upregulated in response to wounding, pathogen infection, and abiotic stresses in numerous plant species (Eulgem et al., 2000; Ulker and Somssich, 2004).

WRKY proteins comprise a large family of zinc finger type transcription factors (Park et al., 2005). The WRKY transcription factor superfamily consists of 74 and 109 members in Arabidopsis and rice, respectively (Eulgem and Somssich, 2007; Ross et al., 2007; Pandey and Somssich, 2009). WRKY proteins contain one or two WRKY domains, which are 60 amino acid regions containing WRKYGQK at the N-terminus, and a $\mathrm{C}-\mathrm{C}-\mathrm{H}-\mathrm{C} / \mathrm{H}$ zinc finger-like motif at the $\mathrm{C}$ terminus (Eulgem et al., 2000; Park et al., 2005). WRKY proteins bind specifically to W-box elements [TTGAC(C/T)] both in vitro and in vivo (Eulgem et al., 2000; Park et al., 2005). All known WRKY proteins contain either one or two WRKY domains. Based on the number of WRKY domains and the zinc finger motif sequence, WRKY proteins can be classified into three distinct groups (I, II, and III). Group I proteins contain two WRKY domains, whereas each of group II and III proteins possess one WRKY domain. Groups II and III differ in the structure of the zinc finger motif. In general, the WRKY domains of group I and II proteins contain the $\mathrm{C}_{2}-\mathrm{H}_{2}\left(\mathrm{C}-\mathrm{X}_{4-5}-\mathrm{C}-\mathrm{X}_{22-23}-\mathrm{H}-\mathrm{X}_{1}-\mathrm{H}\right)$ pattern finger motif, whereas those of group III contain a $\mathrm{C}_{2}-\mathrm{HC}$ motif $\left(\mathrm{C}-\mathrm{X}_{7}-\mathrm{C}-\mathrm{X}_{23}-\mathrm{H}-\mathrm{X}_{1}-\mathrm{C}\right)$ (Eulgem et al., 2000).

In this study, a WRKY transcription factor was cloned from tomato using reversetranscription polymerase chain reaction (RT-PCR) and rapid amplification of cDNA ends (RACE). Furthermore, we characterized its expression patterns under various environmental stresses, such as low temperature $\left(4^{\circ} \mathrm{C}\right)$, salt stress, jasmonic acid, salicylic acid, and Botrytis cinerea Pers. infection. The results showed that LeWRKY1 expression is induced by jasmonic acid (JA), but not by salicylic acid (SA). The majority of the WRKY genes analyzed respond to pathogen attack, and to the endogenous signaling molecule SA (Eulgem and Somssich, 2007; Pandey et al., 2009). LeWRKY1 also responded to pathogen attack (B. cinerea), but not to SA.

\section{MATERIAL AND METHODS}

\section{Plant materials and treatment}

Lycopersicum esculentum Miller seeds were obtained from a local garden and used for experiments shortly after harvest. L. esculentum plants were germinated and grown on MS medium. Seedling treatments were performed as described by Yamaguchi-Shinozaki and Shinozaki (1994) with slight modifications. The seedlings were grown for 3 weeks under photoperiod cycles of $16-\mathrm{h}$ light $\left(26^{\circ} \mathrm{C}\right)$ and 8 -h dark $\left(22^{\circ} \mathrm{C}\right)$. Three-week-old seedlings were treated with JA or SA, or allowed to be infected with $B$. cinerea. Next, they were grown hydroponically in MS solution containing 100 $\mu \mathrm{M}$ JA or $100 \mathrm{mM}$ SA, or allowed to be infected with $B$. cinerea based on the method described by Ferrari et al. (2007). The seedlings were then harvested for RNA preparation. 


\section{Total RNA extraction}

Total RNA was extracted using the UNIQ-10 column Trizol total RNA extraction Kit (SK1321), according to the manufacturer protocol.

\section{RT-PCR and RACE-PCR}

Degenerate forward (5'-TGGMGIAARTAYGGNCARA-3') and reverse primers (5'-TGRBYRTGYTTICCYTCRTAIGTDGT-3') were designed based on the conserved sequence of WRKY transcription factors. Using RT-PCR, a WRKY fragment from L. esculentum (LeWRKY1 fragment) was obtained. The full-length sequence for LeWRKY1 was obtained through RACE-PCR using the 3'-Full Race Cord Set and the 5'-Full Race Cord Set (Takara, Japan). The primers used for 3'-RACE were the 3'RACE adaptor primer and the gene-specific primer 5'-TGGAGGAAGTATGGGCAGAA-3'. The primers used for 5'-RACE were the 5' RACE adaptor primer, 5' RACE outer primer, 5' RACE inner primer (provided by 5' RACE Full kits), a genespecific outer primer (5'-ATCGGCTGGCTGTGGAAG-3'), and a gene-specific inner primer (5'-GGTTCGTGGATGGTTATG-3'). PCR products were fractionated by electrophoresis on $1.2 \%$ agarose gels, and collected using a UNIQ-10 column DNA collection Kit (Shanghai Sangon Biological Engineering Technology \& Services, Co., Ltd., China), after which the fragment was cloned into the pMD19-T vector (Takara, Japan) and sequenced (Shanghai Sangon Biological Engineering Technology \& Services, Co., Ltd., China).

\section{Sequence analysis}

Sequence analysis was performed as previously described (Yang et al., 2003). Sequences were assembled using Seq Man II from DNASTAR, Inc. (Madison, WI, USA). Genes were identified using a combination of several methods. The genes in this region were predicted using GenScan (http:// genes.mit.edu/GENSCAN.html) (Burge and Karlin, 1997). The Arabidopsis settings were chosen for all programs. To identify LeWRKY genes, ClustalX was used to align LeWRKY with identified WRKY genes (Thompson et al., 1997). LeWRKY1 was used for BLASTn and BLASTx homology searches against the GenBank database, which performed as described by Altschul et al. (1997).

\section{Quantitative PCR}

Based on the methods described by Yang et al. (2003), quantitative PCR experiments were performed using an Applied Biosystems ABI 7300 system and SYBR Green I was used as a fluorescent dye to quantify levels of DNA. The $2^{-\Delta \Delta C t}$ method (Livak and Schmittgen, 2001) was used to normalize and calibrate CT values relative to endogenous controls. First-strand cDNA was prepared using the Prime Script ${ }^{\mathrm{TM}}$ RT Reagent Kit (Takara, Japan). To amplify gene-specific products, the following primers were used: LeWRKY1 forward primer: 5'-CAAATGGCTACTTCCTTGACC-3', LeWRKY1 reverse primer: 5'-TGGACTTTTGTTTACCATCTCC-3', actin primers were also used, as previously described by Coker et al. (2005). To validate the quantitative PCR results, each experiment was repeated three times. Results are reported as means of biological replicates with the corresponding standard deviations. Significant differences among different treatments were evaluated using one-way ANOVA ( $P \leq 0.05)$. 


\section{RESULTS}

\section{Cloning of LeWRKY1 cDNA using RT-PCR}

We designed degenerate primers against the conserved sequence of WRKY. Using these primers, we cloned a cDNA fragment. The cDNA fragment was subjected to sequencing and nucleotide BLAST searches, and the results showed that the polypeptide encoded by the CDNA fragment contained a WRKY domain; this was named LeWRKY1.

The full-length cDNA of LeWRKY1 was obtained using RACE-PCR method (accession No. FJ654265). A cDNA clone of approximately $1.7 \mathrm{~kb}$ was sequenced and consisted of an open reading frame beginning with an ATG start codon at position 226 and ending with a TAA stop codon at position 1308. It encoded a 360 -amino acid protein with a predicted molecular mass of $39.77 \mathrm{kDa}$ (Figures 1 and 2). This cDNA clone was designated LeWRKY1. Furthermore, it contained 5' (225 bp) and 3' (312 bp) untranslated sequences. Sequence comparison showed that LeWRKY1 contained one WRKY domain, and it had a $\mathrm{C}_{2} \mathrm{H}_{2}$ type zinc finger domain downstream of the WRKY domain (Figure 2). BLASTp analysis showed that the protein encoded by LeWRKY1 is $\sim 85$ and $79 \%$ identical to CaWRKY (accession No. AAX20040.1) and WIZZ proteins (accession No. BAA87058.1), respectively.

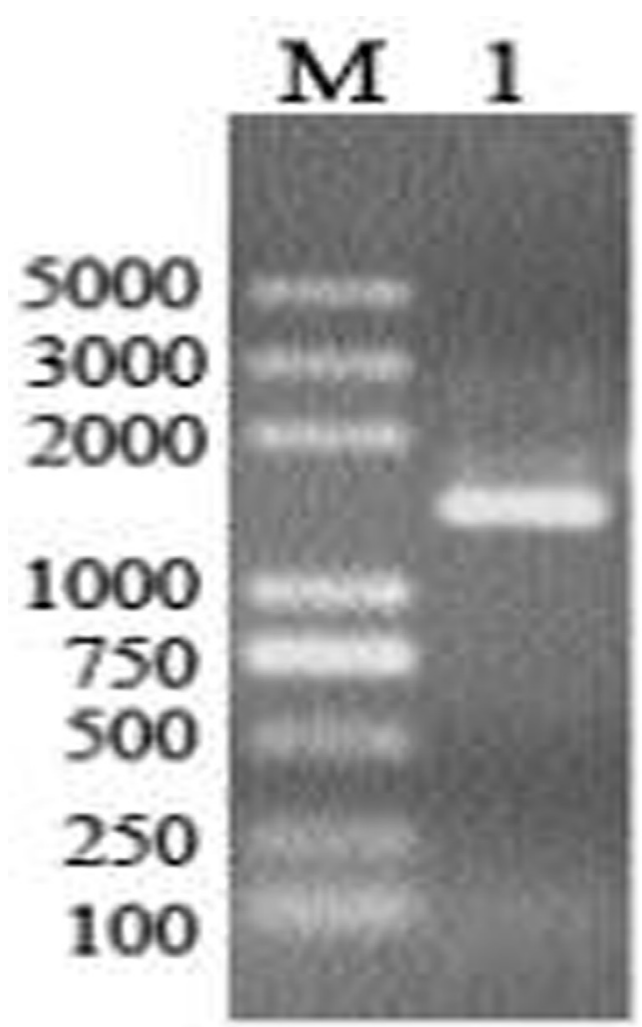

Figure 1. Agarose gel electrophoresis (1.2\% gel) of the full-length cDNA of LeWRKY1 from RT-PCR amplification. Lane $M=$ Trans 2K plus DNA Marker; lane $1=$ LeWRKY1 CDNA. 
I OAOOOTOAOOCAOTOATTCOAOCTOOOIACOCOOCOATCCTCTAGAOATTCTAATACOACTCACTATAOOOCAAOCAO 9 TOOTATOOCAOOTCOACOATTOCOATCATCAOATTTTGAOGAGATAMAAGAAAOTOTTTOTTTCTTTAACTTAAACA

CACCTOAAGAAAGACTACAACTTTOTTGATTCTTGGAATTTTCATACATCAAAGAAGATATTAATTACAATCGAATTT $M E F$

235 ACCAOTITOOTTGATACTTCATTGGATTTOAOCTITAGOCCTCOTCAAAAAGTCCTGAAACAAGAAGTGCAGGGGAT

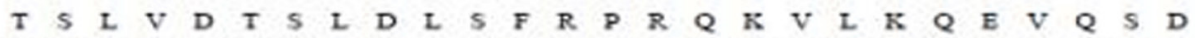

313 TTCACTGGATTGAGCATAGAAAGAGAGATATGOTGGTGAAAATGAGGCAGGGGATTOTTAGAGGACTAAACAGA

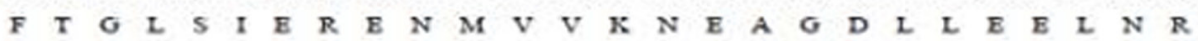

391 GTGAGTAGTGAAACAAGAGTTACAGAGATCTTACTOTAGTTRTGAMATTACAMTOCTTTAGMACCAMATC

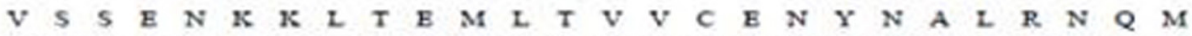

469 ATGGAGTATATGAGCACACANATGGTGTACCTGAAGATACTAGTCCAGGTCAAGGAAMGAAAGCTGANGTATU

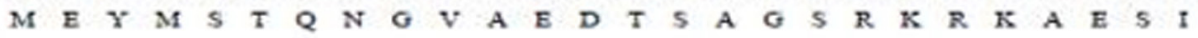

S47 TCTAATCCTGTTAACAACAACAACAATAATAATAACAATATGGATGTTGTTCATGGACGTTCATCAGAAAGCAGTTCO $S N P$ N N N N N N N N N M D V V H G R S $S E S S S$

625 AGTGACGAAGAGTCCTGTTGCAAGAACTCAGAGAGGAGCACATAAAAGCGAAGTTACAATTGTTRCTATGAAGACT S D E E S C C K K I R E E H I K A K V T I V S M K T 703 GATGCATCTGATACCTCTCTIATTGTMAGGATGGTATCAGTGGAGGAGTATGGCCAGAMGTAACTAGAGACAAC

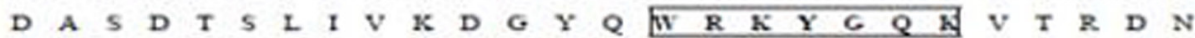

781 CCTIGTCCAGACCTIACTTCAGATCCTCATTTCCACCTGGTOCCCTGTCAMGAMUAGTTCAGAGACCATTGA

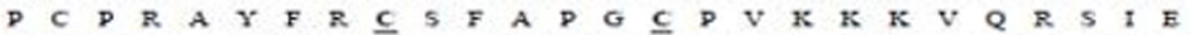

859 GATCAGTCTATTGTGGTAGCAACATATGAAGGAGAACATAACCATCCACGACCTCANACCAGAATCAGGTCCAAGT

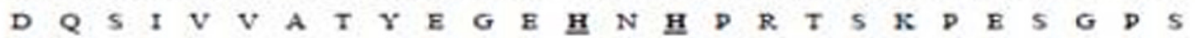

937 ACTATACTTCCACAGCCAGCCGATIMATGTGACACTATCGCGGCACTACTACTTCAGTCOCTTGCTCTACCACT

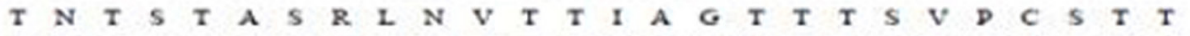

1015 CTCAATTCCTCACGACCMCCATACTCTCGATCTIACTCCACCGMUCAGTGGMUGCGCGATATGMGATGMT I N S S G P T I T L D L T A P K T V E K R D M K M N

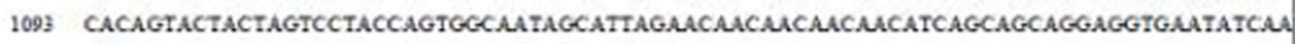
H S T I S D T S G N S I R T T I T I S A A G G E $Y$ S

$117 !$ MTAGGCCAGAGTICANCAGTTCTTGATAGMCMATGGCTACTTCCTGACCMAGATCCMGITTCAMGCACCA $N R$ D E F Q Q F L I E Q M A T S L T K D D S F K A A

1249 CтTGCTOCCGCCATATCAGGAMUTCCTCCMCATATMTCAGACAGOGAGATGOTAUCMUGTCCAGCAGAGC I A A A I $S$ G $G$ K I I $Q$ Q

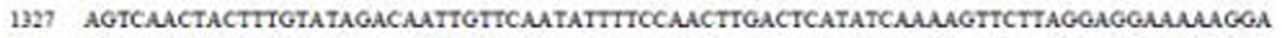

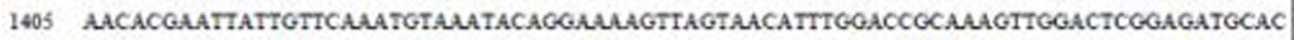

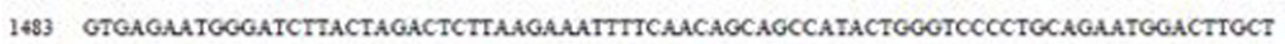

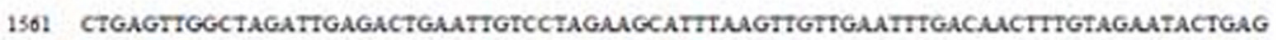

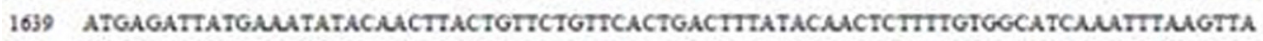

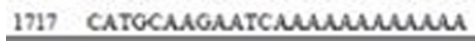

Figure 2. Nucleotide and predicted amino acid sequence of LeWRKY1. The rectangular box shows the conserved amino acid sequence WRKYGQK. The underlined sequence is the $\mathrm{C}_{2} \mathrm{H}_{2}$ zinc finger.

\section{LeWRKY1 expression profiles in different tissues of tomato seedlings}

Expression levels of LeWRKY1 in three tomato seedling tissues (root, stem, and leaf) were measured by qRT-PCR, and data were normalized using LeWRKY1 transcript levels in roots by using the $2^{-\Delta \Delta \mathrm{Ct}}$ method. The relative expression of LeWRKY1 in stems and leaves were 0.89 and 1.6 times that in roots (normalized as 1), respectively (Figure 3 ). 


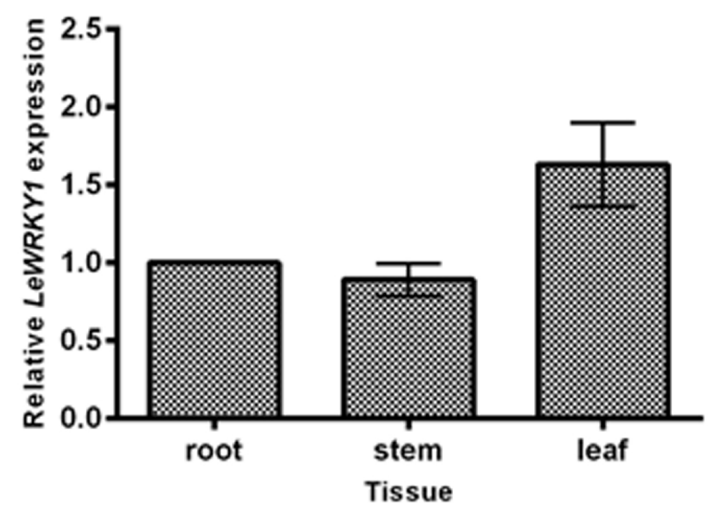

Figure 3. Relative expression of LeWRKY1 in different tomato seedling tissues. Roots, stems, and leaves from threeweek-old seedlings were collected separately, and total RNA was extracted from each tissue for qRT-PCR. Data were normalized to the LeWRKY1 transcript.

\section{Regulation of LeWRKY1 expression by JA and SA in tomato seedlings}

To assess the effects of JA and SA on LeWRKY1 expression, tomato seedlings were treated with $100 \mu \mathrm{M}$ JA or $100 \mu \mathrm{M} \mathrm{SA}$, respectively, for different indicated times (Figure 4). A quantitative PCR experiment using actin as the endogenous control showed that JA could induce LeWRKY1 expression. When treated with JA, statistically significant differences in LeWRKY1 expression were observed. A 50\% increase in LeWRKY1 expression was induced 30 min after JA treatment, and the expression peaked at $12 \mathrm{~h}$ (4-fold increase) (Figure 4a). SA treatment had no significant effect on LeWRKY1 expression (Figure 4b).

a

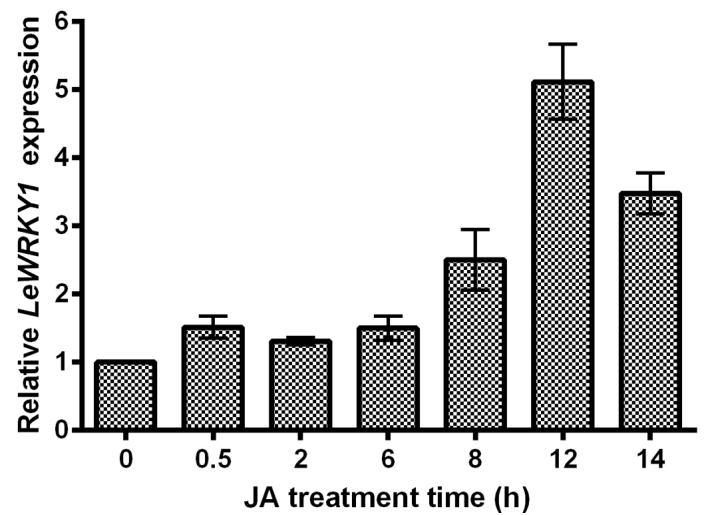

b

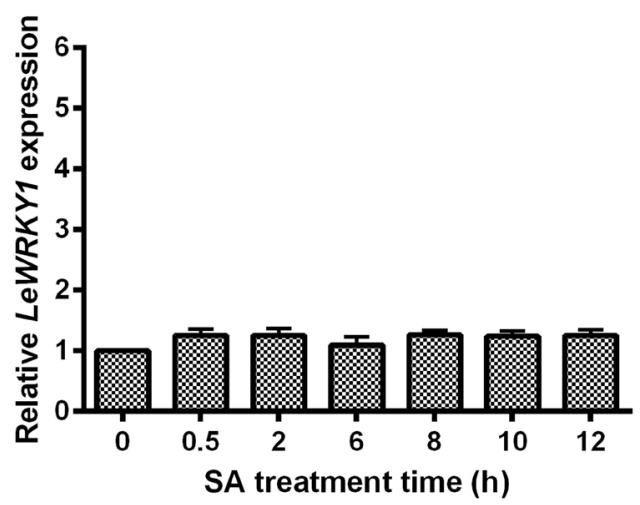

Figure 4. Effect of JA on levels of LeWRKY1 transcription in Lycopersicum esculentum seedlings. a. Seedlings were treated with $100 \mu \mathrm{M} \mathrm{JA}$ for $0,0.5,1,2,6,8,12$, or $14 \mathrm{~h}$. b. The seedlings were treated with $100 \mu \mathrm{M} \mathrm{SA}$ for $0,0.5$, $2,6,8,10$, or $12 \mathrm{~h}$. Actin (U60480.1) was used as the internal control. Data are reported as means $\pm \mathrm{SD}(\mathrm{N}=3)$.

\section{Effect of B. cinerea treatment on LeWRKY1 expression}

LeWRKY1 expression was increased in a time-dependent manner over $48 \mathrm{~h}$ in $B$. cinerea- 
infected tomato seedlings. LeWRKY1 expression increased to about 4.1-fold at $48 \mathrm{~h}$ after treatment (Figure 5). After $72 \mathrm{~h}$, LeWRKY1 expression began to decrease.

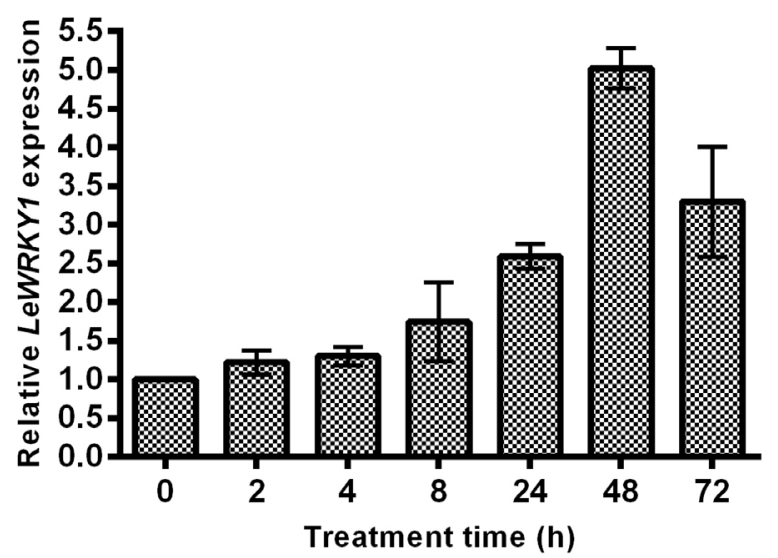

Figure 5. Induction of $L e W R K Y 1$ after Botrytis cinerea infection. The seedlings were treated with $B$. cinerea for $0,2,4$, 8,24 , or 72 h. Actin (U60480.1) was used as the internal control. Data are reported as means $\pm S D(N=3)$.

\section{DISCUSSION}

We cloned WRKY cDNA from tomato, and determined that it encodes the complete amino acid sequence of this transcription factor. The number of WRKY domains and the type of zinc finger motif suggest that LeWRKY1 belongs to group II WRKY transcription factors. LeWRKY1 was detected in the root, stem, and leaf of tomato (Figure 3). LeWRKY is expressed throughout the intact plant, with the highest expression observed in the leaf (Figure 3).

Plants have two interconnected innate immunity pathways. The first involves pathogenassociated molecular pattern (PAMP)-triggered immunity (PTI), which is initiated by the recognition of molecular signatures of many pathogens, and often activates downstream mitogen-activated protein kinase cascades and defense genes. The second involves effector-triggered immunity $(\mathrm{ETI})$, which is driven by plant-disease resistance proteins that directly or indirectly recognize specific pathogen-derived effectors (Chisholm et al., 2006; Pandey and Somssich, 2009). PTI and ETI activate local and systemic defense responses, which are modulated by phytohormones, especially JA and SA (Durrant and Dong, 2004; Bostock, 2005; Pandey and Somssich, 2009). These responses to pathogen attack require large-scale transcriptional reprogramming and include WRKY transcription factors (Eulgem, 2005; Ryu et al., 2006; Naoumkina et al., 2008; Pandey and Somssich, 2009). LeWRKY1 was induced by JA (Figure 4a), but not by SA (Figure 4b). JA-dependent plant defenses are generally activated by necrotrophic pathogens and chewing insects, whereas SA-dependent defenses are often triggered by biotrophic pathogens (Pandey and Somssich, 2009). Synergistic and antagonistic interactions between JA and ethylene signaling have been reported and depend on the stress conditions under investigation (Kazan and Manners, 2008). JA and SA signaling usually act antagonistically, but synergism between these two phytohormones has also been observed (Mur et al., 2006). Our results show that LeWRKY1 can be induced by JA, but not by SA (Figure 4). 
Various plant defense responses against microbial pathogens are regulated by SA and JA pathways (Ferrari et al., 2007). These signaling pathways have been extensively studied, but how the SA, JA, and ET signaling pathways are related to those activated by OGs and other PAMPs remains unknown (Ferrari et al., 2007). B. cinerea infection increased LeWRKY1 expression (Figure 5). Ferrari et al. (2007) indicated that OGs increase Arabidopsis resistance to $B$. cinerea through the activation of defense responses that are independent of SA, JA, and ET, but that SA, $\mathrm{JA}$, and ET are also involved in defense pathways that confer resistance to $B$. cinerea. It is crucial to elucidate the cellular/nuclear components of LeWRKY1 interaction when plants are infected with $B$. cinerea, and to determine how LeWRKY1 is regulated.

\section{Conflicts of interest}

The authors declare no conflict of interest.

\section{ACKNOWLEDGMENTS}

Research supported by the Beijing Natural Science Foundation (\#5132006).

\section{REFERENCES}

Altschul SF, Madden TL, Schäffer AA, Zhang J, et al. (1997). Gapped BLAST and PSI-BLAST: a new generation of protein database search programs. Nucleic Acids Res. 25: 3389-3402.

Bostock RM (2005). Signal crosstalk and induced resistance: straddling the line between cost and benefit. Annu. Rev. Phytopathol. 43: 545-580.

Burge C and Karlin S (1997). Prediction of complete gene structures in human genomic DNA. J. Mol. Biol. 268:7 8-94

Chisholm ST, Coaker G, Day B and Staskawicz BJ (2006). Host-microbe interactions: shaping the evolution of the plant immune response. Cell 124: 803-814.

Coker JS, Vian A and Davies E (2005). Identification, accumulation, and functional prediction of novel tomato transcripts systemically upregulated after fire damage. Physiol. Plantarum 124: 311-322.

Durrant WE and Dong X (2004). Systemic acquired resistance. Annu. Rev. Phytopathol. 42: 185-209.

Eulgem T (2005). Regulation of the Arabidopsis defense transcriptome. Trends Plant Sci. 10: 71-78.

Eulgem T and Somssich IE (2007). Networks of WRKY transcription factors in defense signaling. Curr. Opin. Plant Biol. 10: 366-371.

Eulgem T, Rushton PJ, Robatzek S and Somssich IE (2000). WRKY superfamily of plant transcription factors. Trends Plant Sci. 5: 199-206

Ferrari S, Galletti R, Denoux C, De Lorenzo G, et al. (2007). Resistance to Botrytis cinerea induced in Arabidopsis by elicitors is independent of salicylic acid, ethylene, or jasmonate signaling but requires PHYTOALEXIN DEFICIENT3. Plant Physiol. 144: $367-379$.

Kazan K and Manners JM (2008). Jasmonate signaling: toward an integrated view. Plant Physiol. 146: 1459-1468.

Livak KJ and Schmittgen TD (2001). Analysis of relative gene expression data using real-time quantitative PCR and the $2^{-\Delta \Delta C t}$ method. Methods 25: 402-408.

Mur LA, Kenton P, Atzorn R, Miersch O, et al. (2006). The outcomes of concentration-specific interactions between salicylate and jasmonate signaling include synergy, antagonism, and oxidative stress leading to cell death. Plant Physiol. 140: 249-262.

Naoumkina MA, He X and Dixon RA (2008). Elicitor-induced transcription factors for metabolic reprogramming of secondary metabolism in Medicago truncatula. BMC Plant Biol. 8: 132.

Pandey SP and Somssich IE (2009). The role of WRKY transcription factors in plant immunity. Plant Physiol. 150: 1648-1655.

Park CY, Lee JH, Yoo JH, Moon BC, et al. (2005). WRKY group Ild transcription factors interact with calmodulin. FEBS Lett. 579: $1545-1550$.

Ross CA, Liu Y and Shen QJ (2007). The WRKY gene family in rice (Oryza sativa). J. Integr. Plant Biol. 49: 827-842.

Ryu HS, Han M, Lee SK, Cho JI, et al. (2006). A comprehensive expression analysis of the WRKY gene superfamily in rice plants during defense response. Plant Cell Rep. 25: 836-847. 
Thompson JD, Gibson TJ, Plewniak F, Jeanmougin F, et al. (1997). The CLUSTAL_X windows interface: flexible strategies for multiple sequence alignment aided by quality analysis tools. Nucleic Acids Res. 25: 4876-4882.

Ulker B and Somssich IE (2004). WRKY transcription factors: from DNA binding towards biological function. Curr. Opin. Plant Biol. 7: 491-498.

Yang ZN, Ye XR, Molina J, Roose ML et al. (2003). Sequence analysis of a 282-Kilobase region surrounding the citrus Tristeza virus resistance gene (Ctv) locus in Poncirus trifoliata L. Raf. Plant Physiol. 131: 482-492.

Yamaguchi-Shinozaki K and Shinozaki K (1994). A novel cis-acting element in an arabidopsis gene is involved in responsiveness to drought, low-temperature, or high-salt stress. Plant Cell 6: 251-264. 